\title{
Scrutinizing mammalian reproductive inventions
}

Mammalian reproduction relies on two important evolutionary inventions - lactation and placentation. Two recent papers shed light on how and when these important characteristics evolved.

Most vertebrates are egg laying, and their embryos rely on the egg yolk as a source of nourishment during development. The yolk nutrients are supplied by vitellogenin (VTG), the function of which has been replaced by the placenta and lactation in non-egg-laying mammals. But how did these reproductive innovations evolve?

Taking advantage of existing genomic resources, Brawand et al. used comparative genomics and evolutionary simulations to trace the evolutionary fate of vitellogenin genes (VIT) in mammals. They found that the genomes of placental and marsupial mammals contain only VIT pseudogenes in the regions that are syntenic to those where the three chicken VIT genes lie. They also found that VIT gene function was lost progressively during mammalian evolution - a process that involved multiple independent inactivation events.

Interestingly, monotremes have two VIT genes, a recently inactivated pseudogene and another gene that corresponds to either VIT2 or VIT3 and that is likely to be functional - it shows signatures of purifying selection. Thus, monotremes' intermediate reproductive phenotype - egg laying combined with lactation - is matched at the genomic level.

In mammals, many of the functions of VTG have been taken over by casein, a major component of milk. The authors show that monotremes have three putative casein genes, indicating that these genes emerged in the common mammalian ancestor. By contributing to the nourishment of the young, casein might have rendered some of the VIT genes redundant and this ultimately led to their loss in placental mammals.

Evolution of the placenta, the other major mammalian reproductive innovation, is also likely to have contributed to the relaxation of constraints on VIT genes. In the second paper, Knox and Baker used a time-course microarray analysis of gene expression in mouse and human placenta to explore the mechanisms by which this organ might have evolved.

Their analysis revealed a clear molecular transition mid-gestation - using the Gene Ontology (GO) terms they were able to determine that the first, developing, phase is associated with genes that are mainly involved in growth and metabolism, whereas the mature phase is associated with genes that are involved primarily in steroid metabolism and biosynthesis, angiogenesis, pregnancy and reproduction, and immune-system functions. Having evaluated the evolutionary histories of these placental genes, the authors propose that the evolution of basic placental structures relied on evolutionarily ancient genes, but the involvement of more recently evolved genes in the mature placental stage reveals that duplication and divergence have been crucial for the emergence of the diversity in the length of gestation and litter size seen among placental mammals.

$$
\text { Magdalena Skipper }
$$

ORIGINAL RESEARCH PAPERS Brawand, D. et al Loss of egg yolk genes in mammals and the origin of lactation and placentation. PLoS Biol. $18 \mathrm{Mar}$ 2008 (doi:10.1371/jpiurnal.pbio.0060063) |Knox, K. and Baker, J. C. Genomic evolution of the placenta using co-option and duplication and divergence. Genome Res. 13 Mar 2008 (doi:10.1101/gr.071407.107)

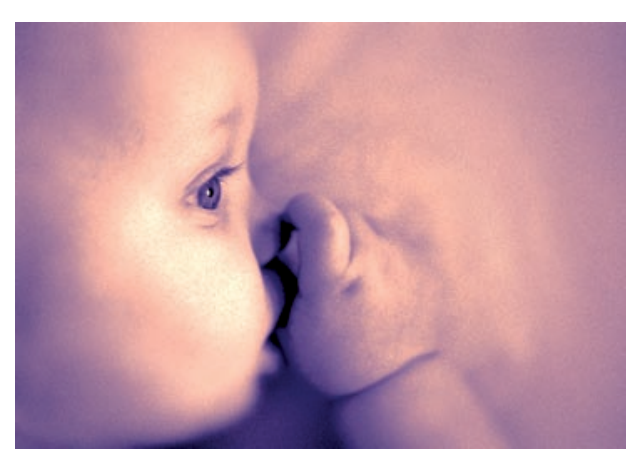

